\title{
Dificultades de la escritura y desaprovechamiento de su potencial epistémico en estudiantes de posgrado
}

Writing Difficulties and Waste of its

Epistemic Potential in Graduate Students

Dificuldades de escrita e desperdício de seu potencial epistêmico em

alunos de pós-graduação

Camilo Giraldo-Giraldo* iD orcid.org/0000-0002-7399-9738

Para citar este artículo: Giraldo-Giraldo, C. (2020). Dificultades de la escritura y desaprovechamiento de su potencial epistémico en estudiantes de posgrado. Revista Colombiana de Educación, 1(80), 173-192.

https://doi.org/10.17227/rce.num80-9633

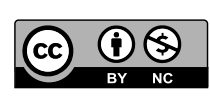

Recibido: 18/02/2019

Evaluado: 23/08/2019

* Magíster en Filosofía. Profesor de la Universidad de Manizales, Colombia. Correo electrónico: cgiraldo@a 


\section{Resumen}

Este artículo de investigación se propone identificar e interpretar las percepciones que tienen estudiantes de posgrado sobre las dificultades en su producción textual y verificar si tanto instituciones como alumnos desaprovechan el potencial epistémico de la escritura (Carlino, 2004; 2013; Cassany y Morales, 2009; Cruz, 2015; Giraldo, 2015; Hernández, 2009; Narvaja, 2009; Ochoa y Cueva, 2017; Ong, 1994; Scardamalia y Bereiter, 1992; Zárate, 2017). El trabajo se realizó en cuatro universidades de Manizales, Colombia. Se trata de un estudio exploratorio de tipo descriptivo para el cual se aplicó una encuesta con 5 preguntas a 80 estudiantes de maestrías y doctorados y a 20 directores de posgrado; además, se hizo entrevista semiestructurada a estos participantes. Se encontró que el 90 \% de los encuestados admite que tiene dificultades para escribir y estas son reiterativas en comparación con otros estudios. Aunque también se halló que el 84 \% dijo conocer las normas de la escritura académica, esto no ha de interpretarse como contradictorio con el resultado anterior, sino como un indicativo de que el conocimiento de los criterios de la escritura científica no es garantía de su aplicación. Así, estudiantes e instituciones deberían procurar, sobre todo, el ejercicio dirigido de la escritura científica. Otro de los hallazgos centrales para este estudio es que el $97 \%$ de los estudiantes manifestó que desconoce la práctica de la escritura con el propósito de aprovechar su potencial epistémico. Se concluye que estudiantes e instituciones generalmente desaprovechan la escritura como instrumento para fortalecer y generar conocimiento.

\section{Palabras clave}

escritura; habilidades; dificultades; experiencia de aprendizaje; estudiantes de posgrado; programas de posgrado

\section{Keywords}

writing; skills; difficulties; learning experience;

postgraduate students; postgraduate programs

\begin{abstract}
The purpose of this research article is to identify and interpret postgraduate students' perceptions about the difficulties in their textual production and verify if both institutions and students are wasting the epistemic potential of writing (Carlino, 2004, 2013; Cassany y Morales, 2009; Cruz, 2015; Giraldo, 2015; Hernández, 2009; Narvaja, 2009; Ochoa y Cueva, 2017; Ong, 1994; Scardamalia y Bereiter, 1992; Zárate, 2017). The work was carried out in four universities in Manizales, Colombia. It is an exploratory descriptive study. The authors administered a 5-question survey to 80 master and doctorate students, and to 20 postgraduate directors plus semistructured interview. The results show that $90 \%$ of the respondents admit that they have difficulties in writing and these are repetitive compared toother studies. Although $84 \%$ of them said they knew the rules of academic writing, it does not contradict the previous result, but suggests that being aware of the criteria of scientific writing is not a guarantee of its application. Thus, students and institutions should seek, above all, the directed exercise of scientific writing. Another central finding of this study is that $97 \%$ of the students said that they do not know the practice of writing to take advantage of its epistemic potential. It is concluded that students and institutions generally do not make the most out of writing as a tool to strengthen and generate knowledge.
\end{abstract}

\section{Resumo}

Este artigo de pesquisa tem como objetivo identificar e interpretar as percepções que os alunos de pós-graduação têm sobre as dificuldades em sua produção textual e verificar se tanto as instituições quanto os alunos perdem o potencial epistêmico da escrita (Carlino, 2004, 2013; Cassany y Morales, 2009; Cruz, 2015; Giraldo, 2015; Hernández, 2009; Narvaja, 2009; Ochoa y Cueva, 2017; Ong, 1994; Scardamalia y Bereiter, 1992; Zárate, 2017). O trabalho foi realizado em quatro universidades em Manizales, Colômbia. Trata-se de um estudo descritivo exploratório no qual foi aplicada uma survey com 5 questões a 80 alunos de mestrado e doutorado e 20 diretores de pós-graduação; além disso, foi realizada uma entrevista semiestruturada com esses participantes. Constatou-se que $90 \%$ dos respondentes admitem ter dificuldades para escrever e estas são repetitivas em relação a outros estudos Embora também tenha sido verificado que 84 \% afirmaram conhecer as regras da redação acadêmica, isso não deve ser interpretado como contraditório ao resultado anterior, mas sim como uma indicação de que o conhecimento dos critérios da redação científica não é garantia de sua aplicação. Assim, alunos e instituições devem buscar, antes de tudo, o exercício direcionado da escrita científica. Outro dos achados centrais para este estudo é que $97 \%$ dos alunos afirmaram desconhecer a prática da escrita para aproveitar seu potencial epistêmico. Conclui-se que alunos e instituições geralmente deixam de perceber a escrita como instrumento de fortalecimento e geração de conhecimento.

\section{Palavras-chave}

habilidades de escrita; dificuldades de escrita; experiência de aprendizagem; alunos de graduação; programas de pós-graduação 


\section{Introducción}

Además de los factores sobre deserción en los posgrados que encontraron investigadores del Banco Mundial para 2017 —por ejemplo, en Estados Unidos se estima en un $25 \%$ y en Colombia en un $37 \%$ (Ferreyra et al., 2017) - la producción escrita también resulta esencial para explicar este fenómeno (Chois y Jaramillo, 2016; Hernández, 2009; Hirschhorn, 2012; Vargas, 2016). Esto porque quienes no logran satisfacer las exigencias de redacción y estructura de los productos escritos posgraduales asumen que ello tiene relación con su capacidad intelectual para el saber disciplinar y desfallecen. Una de las causas es que tanto docentes como instituciones asumen que quienes ingresan a la educación superior cuentan con la competencia escritural correspondiente a su nivel de educación (Cassany y Morales, 2009; Narvaja, 2009) y descuidan el acompañamiento directo de la expresión escrita.

En posgrados, sin embargo, la composición escrita es de alta exigencia puesto que se debe articular con la producción investigativa y la construcción de conocimiento. En este ámbito, el estudiante, con más rigor, debe pasar de ser solo consumidor de escrituras y asumir también el rol de productor; esto es, "de lector a autor en tanto productor de nuevos saberes" (Nothstein y Valente, 2016, p. 129). En síntesis, la formación posgradual es una etapa en la que las prácticas de escritura "no constituyen sólo una instancia de evaluación ni desempeñan exclusivamente una función transmisora de conocimientos, sino que el proceso de escritura es crucial en la elaboración del conocimiento" (Narvaja, 2009, p. 2).

Así, en los estudios de posgrado la escritura está estrechamente relacionada con el saber disciplinar (Carlino, 2013) y con las convenciones de escritura para los productos de esta etapa educativa (artículos para indexar, tesis, informes técnicos). Además, la producción escrita obliga al estudiante de posgrado a considerar estos interrogantes:

\section{¿Cómo se debe presentar una propuesta de elaboración propia, pero sustentada en un soporte teórico pertinente y confiable? y ¿cuáles son los recursos escriturales indispensables que debe manejar según la co- munidad discursiva a la que se dirige y en la que espera insertarse? (Rodríguez y García, 2015, p. 250)}

Lo anterior, porque "la tesis representa evidentemente la exigencia última, sancionada por sustentación o defensa" (Gómez et al., 2010, p. 14).

Por eso, los estudiantes de posgrado "deben desplegar estrategias de sobrevivencia para construir escritos, ya que son éstos la única manera de acreditar determinados cursos" (Cruz, 2015, p. 52); además, en posgrado los "trabajos académicos se caracterizan por una marcada complejidad tanto conceptual como discursiva" (Moya et al., 2017, p. 34), lo que 
significa para el estudiante "enfrentar nuevos retos como la práctica de la escritura y la conceptualización y elaboración de marcos teóricos sólidos" (Moya et al., 2017, p. 37). Entonces, "escribir en el posgrado es una actividad compleja que impone a los aprendices retos de alta envergadura, diferentes de aquellos enfrentados en la educación previa" (Chois, 2016, p. 229). Y en algunas ocasiones "puede parecer que esas normas reducen y hasta constriñen el trabajo y la creatividad tanto del aprendiz como del avezado investigador" (Patiño, 2013, p. xxxı).

Pero, "la imposibilidad de escribir [con determinados requisitos] mina la autoestima y genera sentimientos de culpa" (Ochoa y Cueva, 2017, p. 72), que menguan o impiden una producción escrita acorde con el nivel posgradual. Incluso, luego de que cumplen los demás requisitos muchos no logran culminar la redacción de la tesis o el artículo. Al respecto, Chois y Jaramillo (2016) encontraron que "la complejidad de la escritura en el posgrado podría explicar, por lo menos parcialmente, la baja tasa de graduación en este nivel de formación y el fenómeno conocido como 'todo menos tesis' (тмт)" (p. 230). Así, "para una cantidad significativa de estudiantes la redacción de los trabajos requeridos se convierte en una tarea problemática que obstaculiza, demora y hasta llega a impedir la consecución de sus objetivos de formación" (Narvaja, 2009, pp. 1-2). Por tanto, asumir que quienes llegan al posgrado ya saben escribir con fluidez puede ser fatal para los que enfrentan prácticas discursivas especializadas "que requieren una intervención pedagógica y apoyos específicos" (Hernández, 2009, p. 36) y no lo tienen.

En general, el estudiante llega al posgrado con poca experiencia y muchos prejuicios sobre construir textos académicos; por ejemplo, creer que en investigación existe una única manera correcta y rigurosa de escribir y que se nace con ese talento. O pensar que la escritura potente es propia de unos pocos $\mathrm{O}$, al contrario, que la redacción del trabajo se hace a última hora y "de una sentada". En el primer caso, bloquea la fluidez de la escritura y, en el segundo, la producción escrita resulta ampulosa y vacía, bajo la asunción de que la producción escrita se trata de vaciar unos datos, traer citas entrecomilladas o parafraseadas y no requiere borradores, revisión o reescritura. Los dos extremos representan motivos para que el estudiante al final no logre la producción escrita o desista de graduarse.

Por eso, en la formación posgradual suelen enfrentarse dificultades para "la comunicación de propuestas o hallazgos de investigación [que] exigen construir textos cohesivos, coherentes, adecuados y originales para difundir sus aportaciones" (Rodríguez y García, 2015, p. 251). Y aunque sobre el asunto "existen pocos desarrollos teóricos, metodológicos y empíricos" (Vargas, 2016, p. 101), el presente trabajo tiene antecedentes diversos. Por ejemplo, para estudiantes de posgrado en Malasia la finalización oportuna de la tesis o los artículos constituye una barrera Joseph, 
2018). Los retos básicos de estudiantes de posgrado, según el estudio de Joseph (2018) en posgrados de Malasia: "El primer desafío que los estudiantes describieron fue captar ciertas habilidades, competencias y entendimientos que a menudo se esperan de los estudiantes a nivel de posgrado" (p. 33). En la Universidad de Virginia, Estados Unidos, Myers (1999) realizó un estudio para examinar las razones por las que desertaron candidatos a doctores. Al preguntar a los 11 candidatos a doctor, los motivos que expusieron para no completar la tesis fueron "la frustración y/o la pérdida de interés ocuparon el primer lugar (36\%), seguidas por consideraciones financieras (27\%), circunstancias familiares $(18 \%)$ y situaciones de tiempo y apoyo (9\%, cada uno)" (Myers, 1999, pp. 60-61).

En un estudio que aplicó en la Universidad Pedagógica Nacional de México, Cruz (2015) diagnosticó las dificultades discursivas y lingüísticas en torno a la producción escrita en estudiantes de posgrado; encontró complicaciones para reconocer el tipo de texto académico y su estructura y las normas de producción textual; no saber cómo empezar o jerarquizar ideas, fallas ortográficas y sintácticas y desconocimiento de conectores para enlazar frases y párrafos. También en México, Márquez et al. (2013) hicieron un estudio que partió de la pregunta ¿Cuáles son las habilidades de redacción académica que presenta un grupo de alumnos de la materia Proyectos 1 de una maestría en Educación en una modalidad a distancia? Los autores hallaron "dificultades en el manejo de la redacción técnica y de la estructura propia de artículos y tesis; también se encontró que se presentaron ideas en el texto sin acreditar a los autores de las ideas expresadas" (p. 13). Es decir, problemas lingüísticos como la aplicación de la ortografía y la sintaxis, la estructura del párrafo, y discursivos como el manejo de citas y referencias y la concisión y precisión discursiva textual. Concluyeron que "al haber evaluado los documentos de los alumnos y contrastándolos con las características correspondientes a la redacción académica: se consideran regulares o malas" (Márquez et al., 2013, p. 15). $\mathrm{Y}$ al indagar por la percepción que tienen los docentes sobre la escritura de los estudiantes, los investigadores hallaron que "Los profesores tutores entrevistados inicialmente describieron las habilidades de Redacción Académica de los alumnos como regulares o malas, así, coinciden con la información derivada del Diagnóstico realizado" (Márquez et al., 2013, p. 1).

Otro estudio en una maestría de México es el de Hernández (2009), que busca describir y analizar las dificultades en el proceso de escritura de los estudiantes. En este se recolectó un listado de obstáculos que expresaron los maestrandos al momento de escribir su tesis y que se pueden agrupar así:

1. Convenciones de los textos académicos: género y formato (naturaleza expositiva o argumentativa de la escritura académica, formato, uso de citas y referencias, etcétera).

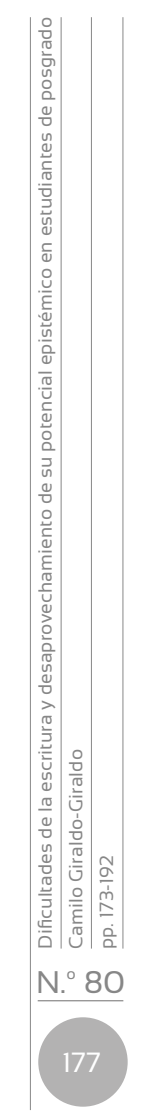


2. Estrategias de escritura (planeación, revisión).

3. Convenciones y habilidades básicas de lectura/escritura (ortografía y puntuación, sintaxis, redacción), así como estilo o registro (coloquial, formal).

4. Conocimiento previo y/o comprensión de lectura.

5. Posición o postura personal (ausencia de punto o argumento: lo que ellos quieren decir) (p. 24).

Ahora bien, en Argentina, Carlino (2005b) hizo un estudio sobre las vivencias de 41 sujetos: 23 posgraduados que han obtenido su título de magíster y 18 maestrandos que tienen sus tesis en curso, de universidades de diversas provincias. Frente a la pregunta sobre los obstáculos en la elaboración de la tesis, evidenció dificultades referidas directamente a la producción textual como "problemas para acotar el tema y plantear el problema de investigación, dificultad al escribir, falta de autoconfianza, dificultad de acceso a bibliografía, dificultad en recolección de datos" (Carlino, 2005b, p. 417). También halló percepciones desfavorables frente a la escritura de la tesis como un "un gran esfuerzo o desafío; señalan que han pasado por momentos de 'saturación', 'hastío' o 'intranquilidad', sintiendo la tesis como una obligación que los agobia" (Carlino, 2005b, p. 417). Y señala otras percepciones de los encuestados sobre escribir su tesis, frente a lo cual expresaron sentimientos de "tensión", "malestar", "amor/odio", "lucha con uno mismo".

En Colombia, Ochoa y Cueva (2017) indagaron por las causas del bloqueo para escribir la tesis en estudiantes de posgrado; hallaron que de 66 estudiantes, 16 no finalizaron la tesis por dificultad para la escritura y que de 23 que sí la concluyeron, dijeron no haber disfrutado la escritura de su trabajo y ejemplifican con estos testimonios: "Al iniciar es el miedo a escribir, a conectar todo con coherencia y cohesión. A involucrar las referencias y a pensar en la metodología" "Pasé seis meses luego de haber terminado la recogida de datos sin poder escribir ni una sola palabra, literalmente" (p. 72). También expusieron motivos personales ("perfeccionismo, ansiedad, desazón frente a un trabajo que es muy complejo, falta de relación entre la tesis y el campo laboral del tesista, expectativas demasiado altas frente a la tesis, falta de seguridad frente al proceso de escritura, frustración" [p. 73]), académicos ("las causas se relacionan con el tesista, con la tesis y con el director de la tesis [...] Desconocimiento del tema o de lo que debe hacerse [...] poca formación en escritura y en investigación [...] el manejo de las voces" [p. 74]) e institucionales ("los desacuerdos entre profesores de seminario y director de tesis, la falta de líneas de investigación y la falta de reglas claras sobre la tesis y su evaluación" [p. 75]).

Vargas (2016) en un estudio de caso que realizó en una universidad pública colombiana analiza las representaciones sobre la escritura académica que tiene una estudiante de maestría. Encontró que la participante 
tenía habilidad para escribir relatorías y, en alguna medida, ensayos académicos, pero que, según expresó ella, esto no la capacita para escribir su trabajo de grado. Además, "Ella sostiene que no existe una enseñanza explícita de la escritura académica en el nivel del pregrado y mucho menos en el nivel del posgrado" (p. 121), aunque debería haberlo, según la participante en ese estudio.

En general, entonces, las dificultades para la producción textual en estudiantes de posgrados atienden, principalmente, a "aspectos como los textos académicos (naturaleza expositiva o argumentativa de la escritura académica, formato, uso de citas y referencias, etcétera) y estrategias de escritura (planeación, revisión)" (Hernández, 2009, p. 24), además de los atenuantes personales e institucionales. Sin embargo, los estudios sobre las dificultades para la producción escrita en estudiantes de posgrado aún escasean. En un estado del arte de Chois y Jaramillo (2016) acerca de escritura en posgrado en idioma español reúnen 39 investigaciones acerca de las condiciones que, generalmente, inciden en la elaboración de una tesis; algunos de ellos describen diagnósticos y necesidades de los procesos de escritura. Pero ninguno apunta a revisar, además de las dificultades de la escritura, el aprovechamiento o no del potencial epistémico de la escritura, como lo plantea el objetivo del presente estudio: identificar e interpretar las percepciones que tienen estudiantes de posgrado sobre las dificultades en la producción textual y verificar si las instituciones desaprovechan la escritura como instrumento para aprender.

Ahora, particularmente, la atención institucional a los problemas de la composición escrita de estudiantes de posgrado aún es una tarea pendiente. "El tema de la escritura en el contexto docente e institucional no rebasa la frontera de pre-ocuparse a ocuparse del problema" (Cruz, 2015, p. 51). Y al decir de Rodríguez y Leal (2016) en México, por ejemplo, "no todos los posgrados integran mecanismos didácticos y/o curriculares para apoyar a sus alumnos en los procesos de escritura académica aunque hay acciones individuales que reflejan el interés de los profesores universitarios por abordar estos temas" (pp. 226-227). Para el caso de Colombia,

No existen políticas y diseños institucionales fuertes en los cuales la producción de conocimiento y la escritura académica estén articuladas. En otras palabras, la escritura académica no ocupa un lugar transversal en los currículos de los posgrados, a pesar de su potencial epistémico. (Vargas, 2016, p. 101)

No obstante, "a pesar de ser una problemática detectada y de que se ha conocido su repercusión en la calidad de los programas y de quienes se gradúan, son escasas las iniciativas o muy pocas las propuestas de acciones concretas, en el nivel superior, que puedan ayudar a resolverla" (Franco, 2016, p. 152). Es común, entonces, que las necesidades de apoyo 
que tienen estudiantes de posgrado para la producción escrita sean de orden conceptual o se relacionen con los métodos de investigación. Y es que en el proceso de escritura, por lo general, las pautas que se entregan a los estudiantes "tienden a ser únicamente esquemas muy generales, de tipo formal o estructural, lo que permite inferir que se presupone en ellos una pericia escritora que los habilita para trabajar en forma independiente" (Narvaja, 2009, p. 4). Es decir, los programas de posgrado asumen, una habilidad consolidada para la escritura en quienes ingresan.

En cuanto al aprendizaje disciplinar y la producción intelectual, cuando se usa la escritura conscientemente como instrumento fortalece conocimiento, permite pensar y hallar ideas, y crear o consolidar comprensión temática porque "cuando se escribe afloran las debilidades y fortalezas sobre la comprensión de un tema. Mediante la redacción, el autor-investigador distingue con más facilidad las ideas relevantes de las secundarias" (Giraldo, 2017, p. 13). Por eso, además de inquirir acerca de las percepciones de los participantes sobre los problemas para la escritura, este estudio también sondea si los estudiantes e instituciones aprovechan la composición escrita como herramienta para pensar y construir conocimiento.

Este trabajo se justifica, entonces, porque busca visibilizar problemas para la composición escrita en estudiantes de posgrado y relaciona el aprovechamiento del potencial epistémico de la escritura con la calidad de los programas académicos y con el fortalecimiento del aprendizaje disciplinar de los estudiantes. Es decir, muestra un uso de la escritura que va más allá del medio para transmitir lo ya construido, lo ya pensado, las ideas listas y, en esa medida, como un objeto desvinculado de la producción de conocimiento (Pérez y Rincón, 2013). Lo que defiende, empero, este trabajo, es precisamente que escribir en la universidad, sobre todo en posgrados, es una actividad que "está relacionada de modo específico con cada disciplina, ya que cada una de ellas tiene convenciones y normas de escritura distintas" (Franco, 2016, p. 153). Se trata, pues, de que la escritura se asuma en los posgrados como un instrumento con potencial epistémico; una herramienta para detectar falencias y fortalezas o profundidad y consistencia sobre un saber disciplinar. Y, siguiendo a Ong (1994), como la escritura reestructura la conciencia su práctica implica crecimiento individual que, a la postre, beneficia la triada sujeto-institución-entorno.

Por eso, complementa esta investigación la perspectiva teórica que asume la escritura como una expresión que trasciende la función de registrar información o conocimiento o de servir únicamente para plasmar lo pensado, pues la composición escrita es un instrumento para desarrollar pensamiento y generar saber disciplinar (Carlino, 2004, 2013; Cassany y Morales, 2009; Cruz, 2015; Giraldo, 2015, Hernández, 2009; Narvaja, 2009; Ochoa y Cueva, 2017; Ong, 1994; Scardamalia y Bereiter, 1992; Zárate, 2017). Ese contexto teórico se particulariza, por ejemplo, en la 
corriente "Escribir a través del currículo" (Carlino, 2004a; Marinkovich y Morán, 1998), la cual se funda en que "la sistematización de la producción escrita no sólo es parte integrante de la asignatura de castellano o lenguaje, sino también de otras disciplinas en que la escritura constituye un instrumento afianzador del aprendizaje" (Marinkovich y Morán, 1998, p. 1).

Otra iniciativa inserta en la perspectiva de la escritura como herramienta con potencial epistémico es el movimiento "Escribir a través del currículum": surgió durante la década de los ochenta; sostiene que la escritura es fundamental para aprender y propone usarla en las clases de todas las disciplinas. "En algunas instituciones surgen dos variantes de la teoría y práctica de este enfoque: una 'escribir para aprender' y otra 'escribir en las disciplinas', designados como 'cognitivo' y 'retórico', respectivamente" (Marinkovich y Morán, 1998, p. 4). Según este enfoque teórico, "Escribir es un método para pensar. En consecuencia, no puede enseñarse fuera del abordaje de los temas que los alumnos han elegido estudiar" (Carlino, 2004a, p. 2); es decir, que sea una práctica académica transversal.

Incluso, desde esta perspectiva una de las estrategias recomendadas para la calidad educativa es aprovechar mejor la expresión escrita. Para ello, es vital que la escuela procure aplicar "la premisa de que es necesario ayudar a los estudiantes a pasar de procesos de composición que consisten en 'decir el conocimiento' a procesos de composición que consisten en 'transformar el conocimiento'" (Scardamalia y Bereiter, 1992, p. 45). En este trabajo, tal postura representa la clave para superar la problemática, puesto que se anima a considerar la escritura como proceso del pensamiento reflexivo y crítico y, ante todo, como instrumento que potencie el aprendizaje y la generación creativa de conocimiento.

Así pues, el objetivo de este estudio es identificar e interpretar las percepciones que tienen estudiantes y directores de posgrado sobre las dificultades en la producción textual y verificar si las instituciones desaprovechan la escritura como instrumento para aprender. La investigación se aplicó en cuatro universidades de Manizales, Colombia, en posgrados de Ciencias Sociales y Humanas; se consultaron 80 estudiantes y 20 directores de posgrado.

\section{Metodología}

Esta investigación es de tipo mixto pues combina dos perspectivas: exploratoria y descriptiva. En ese sentido, se crearon instrumentos con el fin de recopilar la información que permitiera describir e interpretar las percepciones de estudiantes y docentes sobre la escritura en posgrados. Esto, con el fin de atender a las dos cuestiones que plantea el objetivo: 1) conocer la percepción que tienen los participantes sobre dificultades en la producción 
textual y conocer percepciones de directores de posgrado alrededor del asunto, y 2) indagar si estudiantes e instituciones están desaprovechando el uso de la escritura como una herramienta con poder epistémico.

Para lo anterior, se diseñaron dos instrumentos de consulta: uno para estudiantes y otro para directores de posgrado. Antes de su aplicación, se entrevistó a dos directores de posgrado para conocer su opinión sobre los instrumentos, quienes hicieron sugerencias para ajustar el contenido. Luego, se envió a los estudiantes de maestría y doctorado de las cuatro universidades — con la ayuda de los responsables de cada programa académico — un correo en el que se invitaba a responder la encuesta que se dispuso en línea a través de Google Drive. Por este medio respondieron 51 estudiantes (45 de maestría y 6 de doctorado), y a 29 más (21 de maestría y 8 de doctorado) se les aplicó la encuesta de manera personal, con el fin de ajustar la muestra con 80 participantes (maestría 66 y doctorado 14). En el caso de los directores de posgrado, a 20 se les hizo la encuesta y una entrevista semiestructurada de forma personal ( 15 de maestría y 5 de doctorado).

Las encuestas se conformaron con preguntas cerradas (Sí/No) y abiertas (¿Por qué?, ¿Cómo?) 5 en el primer caso y 4 en el segundo. Para este estudio no se consideró relevante preguntar por el género o la edad de los participantes. Los datos básicos fueron el nivel de posgrado y el área del mismo y las preguntas centrales referidas al objetivo de esta investigación, tal como muestran los resultados.

Este método descriptivo se adecuó a este trabajo por cuanto "busca especificar las propiedades, las características y los perfiles de personas, grupos, comunidades, procesos, objetos o cualquier otro fenómeno que se someta a un análisis" (Hernández et al., 2010, p. 80). Tal camino, como lo recomiendan Hernández et al. (2010), resultó útil para mostrar algunos ángulos o dimensiones del fenómeno; ello implicó su caracterización y el surgimiento de interpretaciones que aporten al conocimiento del asunto, ya que, como afirman Ibáñez y Marín (2008), el método descriptivo también sirve como "un conjunto de tácticas que se emplean para constituir conocimiento" (p. 6). Esto, sin la pretensión de generalizar los resultados que se obtuvieron de estudiantes y docentes de las cuatro universidades de Manizales, en las que se realizó el estudio.

\section{Resultados}

A continuación se presenta el consolidado de respuestas para cada encuesta, la de estudiantes y la de docentes (tabla 1), y se exponen comentarios de los estudiantes en el orden como aparecen en la tabla y se cotejan con las de los docentes en la medida en que guardan relación. Luego, en ítem aparte, se presenta la discusión en torno a los hallazgos. 
Sobre la primera pregunta a los estudiantes de posgrado, la expectativa de este trabajo era que la mayoría declarara que desconoce los requisitos para elaborar la tesis y los artículos científicos; pero solo el $16 \%$ de los consultados se expresó en este sentido: de los 80 encuestados, el $84 \%$ (67), afirmaron conocer cómo se hace la tesis. Este aumento en el número de estudiantes que saben cómo elaborar la tesis y los artículos según requisitos de indexación puede ser explicable por el acceso a internet, por ejemplo. Varios de los consultados indicaron que conocen o actualizan los requisitos en sitios de esta red; sobre todo quienes cursan doctorado manifestaron alguna experiencia: "Trabajo como docente investigador hace diez años y tengo cierta experticia al respecto" o "El conocimiento lo he obtenido con la práctica, además de asesorar trabajos investigativos o de tesis". Otros testimonios fueron: "Se supone que un estudiante de posgrado debería saber hacerlo", "No es competencia de la institución, es un tema más de ser autodidacta", "Creo que ya en posgrado debemos saber cómo escribir" y "Es responsabilidad de cada persona. Supongo que se aprende leyendo y escribiendo".

En consonancia con este hallazgo, el 90 \% de los directores de posgrado respondieron en la pregunta 1 (véase la tabla 2), que la institución da por sentado que los aspirantes a los posgrados deben conocer los criterios de la escritura académica y las normas para citar. Uno de los testimonios de los directores de posgrado indica que "Al aspirante se le pide un escrito, además de la entrevista, en el cual debe sustentar su conocimiento conceptual básico de la disciplina con una redacción adecuada, pero no evaluamos competencia escritural". Los dos directores que contestaron afirmativamente, explicaron que la exigencia se hace a través de un escrito en el que el estudiante debe mostrar el dominio básico conceptual de la disciplina, además de rigor ortográfico, sintáctico y semántico; "la propuesta debe ser contextual, coherente y consistente y mostrar criterios de escritura académica con las respectivas citas y referencias. Esa parte la valoramos al menos en un 30 \% del trabajo", explicó uno de ellos.

\section{Tabla 1.}

Consolidado de respuestas cerradas de la encuesta a estudiantes de posgrados

\begin{tabular}{llll}
\hline & Preguntas & \multicolumn{2}{c}{ Respuesta } \\
\cline { 2 - 3 } & & Sí & No \\
\hline 1. ¿Conoce los requisitos de escritura para tesis y artículos científicos? & 67 & 13 \\
\hline $\begin{array}{l}\text { 2. Al escribir la tesis o el } \\
\text { artículo, usted siente: }\end{array}$ & Incapacidad y frustración & 48 & - \\
\cline { 2 - 3 } & Motivación y gusto & - & 32 \\
\hline 3. ¿Tiene dificultades para escribir la tesis o el artículo científico? & 72 & 8 \\
\hline
\end{tabular}




\begin{tabular}{ccc}
\hline & Preguntas & \multicolumn{2}{c}{ Respuesta } \\
\cline { 2 - 3 } & Sí & No \\
\hline 4. ¿Espera que la institución le enseñe cómo escribir la tesis o el artículo? & 35 & 45 \\
\hline $\begin{array}{l}\text { 5. ¿Sabe usar la escritura como instrumento de aprendizaje, para pensar } \\
\text { o crear y no solo como medio de transmisión de lo ya pensado? }\end{array}$ & 76 & 4 \\
\hline
\end{tabular}

Fuente: elaboración propia.

\section{Tabla 2.}

Consolidado de respuestas cerradas de la encuesta a directores de posgrados

\begin{tabular}{lcc}
\multicolumn{1}{c}{ Preguntas } & \multicolumn{1}{c}{ Respuesta } \\
\cline { 2 - 3 } & Sí & No \\
\hline $\begin{array}{l}\text { 1. ¿Debe el estudiante acreditar fluidez para la } \\
\text { escritura académica al ingresar al posgrado? }\end{array}$ & 2 & 18 \\
\hline $\begin{array}{l}\text { 2. ¿Tiene el posgrado actividades de capacitación } \\
\text { para los estudiantes en escritura académica? }\end{array}$ & 5 & 15 \\
\hline $\begin{array}{l}\text { 3. ¿Tienen los estudiantes una guía para la redacción y el } \\
\text { cumplimiento de normas para la tesis y los artículos? }\end{array}$ & 3 \\
\hline $\begin{array}{l}\text { 4. ¿Promueve entre los docentes el uso de la escritura } \\
\text { como apoyo para consolidar saber disciplinar? }\end{array}$ & 4 \\
\hline
\end{tabular}

Fuente: elaboración propia.

Frente a la pregunta 2, 48 estudiantes manifestaron incapacidad y frustración al escribir la tesis o el artículo, mientras que 32 dijeron no sentir motivación y gusto. Es decir, el 60 \% de los entrevistados podría estar en peligro de no terminar el ciclo de estudio posgradual a causa de sus dificultades para escribir la tesis. Esto podría tener relación con la pregunta 3, en la que 72 estudiantes, el $90 \%$, dijeron tener problemas para escribir la tesis o el artículo, a pesar de que en la primera pregunta muchos de ellos contestaron conocer las normas y requisitos para la escritura académica. Parece una contradicción; sin embargo, de esta situación se puede deducir que conocer los requisitos no garantiza la habilidad para escribir la tesis o los artículos con criterios de indexación.

De todas formas, en el caso de este trabajo, es alto porcentaje de estudiantes que manifiestan desagrado o incapacidad y frustración para elaborar la tesis. Algunas de las razones que esgrimieron se relacionan con el acompañamiento de los directores de tesis: 22 de los encuestados expusieron como causa de su angustia la falta de compromiso del director de tesis, "pues no colaboran con indicaciones para escribir y porque dilatan la revisión de los avances, lo que hace perder ritmo en la escritura", expresó uno de los participantes. Este resultado contrasta con el estudio de Hirschhorn (2012) 
en el que el 84 \% de los estudiantes de posgrado que consultó en Argentina se mostró satisfecho con la realimentación del director en cuanto a la redacción del trabajo. De todas formas, "Lo complejo es que la tesis es la interacción de los [dos] actores y sus motivaciones" (Gómez et al., 2010, p. 14), sobre todo en un proceso como el de la escritura.

Sobre el papel de los directores de tesis, sin embargo, aún son pocas las investigaciones (Chois y Jaramillo, 2016), a pesar de que es un factor clave en la formación del estudiante y su culminación del posgrado. Incluso, según los resultados de este estudio en particular, las instituciones no contemplan una actualización del docente de posgrado en escritura académica para que asesore mejor la del estudiante.

Ahora, en los resultados de la pregunta 4 sobre si espera que la institución le enseñe cómo escribir, solo 35 de los 80 encuestados (44%) espera que la institución sea parte de la solución, lo que puede resultar contradictorio, ya que el 90 \% manifestó tener dificultades. Aquí podría ocurrir que los estudiantes de posgrado prefieren la asesoría externa para elaborar o corregir su tesis y artículos, precisamente por la poca presencia de la institución en este asunto. Incluso, las preguntas 2 y 3 a los directores evidencian que en los programas de posgrado que se consultaron en las cuatro universidades es escasa la intervención institucional para fortalecer la escritura de los estudiantes: respecto de las actividades de capacitación en escritura académica, 5 de los 20 directores de posgrado afirman que las tienen; y frente a la existencia de guías para redactar la tesis y los artículos, 3 de los 20 directores consultados dijeron que el programa las ha planteado.

En relación con la pregunta 5 a los estudiantes sobre el uso de la escritura como instrumento de aprendizaje, 76 de 80 estudiantes (el $95 \%$ ) manifestaron que desconocen esta aplicación de la escritura, en tanto que 4 de los 20 los directores de posgrado respondieron que los programas que dirigen no promueven ni en estudiantes ni docentes el uso de la escritura como apoyo para consolidar saber disciplinar o como potencial epistémico. El $80 \%$ de los directores encuestados asegura que no es necesario, en el caso de los docentes, instruir en torno a la escritura académica toda vez que se asume su fluidez desde el ingreso al programa, lo mismo que se espera de los estudiantes: "El profesor de nuestro posgrado debe acreditar artículos publicados y debió haber realizado una o dos tesis previamente, lo que demuestra su capacidad para asesorar". Otro de los directores expresó que "los docentes de posgrados pueden participar de actividades académicas que ofrece esta universidad para todos sus profesores sobre la escritura académica, pero nuestro programa no ha contemplado esa posibilidad".

En general, pues, las percepciones que expresaron los estudiantes sobre las dificultades para la escritura son del orden psicológico, de condiciones personales y de habilidad discursiva. Esta última, la perti- 
nente a este trabajo, es la más generalizada y para la cual se presentan razones diversas: "Me es difícil empezar a redactar", "Siento que en ocasiones soy muy pobre en mi léxico y ordenar las ideas se me dificulta, por ese motivo escribir me es complicado", "No conozco el manejo del lenguaje científico de las revistas y ello me impide avanzar o empezar el trabajo escrito" $y$ "Tiendo a usar algo de vocabulario informal y suelo preocuparme mucho sobre qué autores citar", fueron algunos de los testimonios. En ese sentido, Gómez et al. (2010) encontraron que los grandes problemas para elaborar la tesis se resumen en dos aspectos "encontrar el tema de la tesis y la habilidad práctica para la escritura" (p. 14). No obstante, en este estudio algunos de los consultados refirieron problemas psicológicos o personales mediante expresiones como "Escribir es lo más complicado, enfrentarse a escribir es difícil", "Aplazar la escritura es la salida a la angustia que ésta me produce", "Me angustia el tiempo de entrega y ello me bloquea".

\section{Discusión}

La mayoría de las dificultades que manifiestan los participantes en este estudio se asemejan a las identificadas en otros trabajos. Por ejemplo, Ochoa y Cueva (2017) encontraron que los bloqueos de los estudiantes de posgrados para escribir, generalmente, se deben más a factores cognitivos que emocionales. Y "lo cognitivo hace referencia a los conocimientos sobre la escritura, las reglas lingüísticas, estilísticas, sociolingüísticas y retóricas, los procesos de planificación, los esquemas discursivos y las actitudes hacia la escritura" (p. 65).

Por eso, el escaso acompañamiento de la institución para ayudar a fortalecer la capacidad para escribir podría significar decepción para algunos estudiantes, uno de los motivos de deserción. Esto podría ocasionar que busquen la ayuda de personas expertas en hacer el trabajo, lo que afecta la oportunidad de que el estudiante obtenga un nivel adecuado de conocimiento. Es más, en casos extremos, aquellos que están solos (por la poca presencia del director de tesis o de acompañamiento institucional) podría recurrir al plagio tomando páginas o capítulos de trabajos publicados. Como se observa en este estudio, de los 80 estudiantes consultados, 35 (el $44 \%$ ) dijeron que la institución debería acompañar la escritura de la tesis y los artículos. Aunque, en este caso, no parece un porcentaje alto el que lo reclama, es un punto que debería suscitar reflexión, ya que la calidad de los trabajos de los estudiantes de posgrado también dice de la calidad de la institución y, por ende, del programa. Al respecto, para Muñoz et al. (2016) en la escuela hay una flexibilidad extrema en cuanto al manejo de los criterios en la producción textual. Es decir, cierta permisividad o desgano para controlar la calidad de los trabajos escritos. 
Ahora bien, en relación con la pregunta que plantea el uso de la composición escrita como un instrumento con potencial epistémico el resultado muestra que la mayoría de los estudiantes consultados (el $95 \%$ no conocen esta aplicación de la escritura. Los 4 que respondieron que conocen y practican la escritura como herramienta de aprendizaje expusieron razones como "Porque para mí la escritura es la manera en que traduzco mis aprendizajes, hallazgos, al tiempo que corrijo, aprendo y resignifico los procesos" y "Sí; porque al momento de escribir también se aprende, se construye un mundo propio, además es la posibilidad de crecimiento continuo y no simplemente redactar". Dos de ellos se identificaron como docentes en ejercicio y conscientes de esta perspectiva de la escritura que, de hecho, practican con sus estudiantes: "He puesto en práctica la escritura para el aprendizaje en mis espacios de enseñanza, dejando resultados favorables en los aprendices". Sin embargo, son casos puntuales de una minoría que frente al número que acude a los estudios de posgrado invita a pensar sobre el papel de los programas, y de las universidades, en animar formas de aprendizaje autónomo o maneras de que los estudiantes apropien y aumenten niveles de conocimiento.

Por lo anterior, como lo mostraron los resultados de este estudio, el $80 \%$ de los directores de posgrado asume que los docentes, dada la trayectoria en la producción escrita que se les exige, están en capacidad de asesorar a los estudiantes. Sin embargo, la objeción es que así los docentes tengan publicaciones de altos niveles y estén capacitados en producción escrita, en el estilo docente aún predomina la transmisión de contenidos para los cuales el tiempo está cronometrado. Por eso, "el debate se ha desplazado desde la discusión sobre si es apropiado o no ocuparse de la lectura y escritura en los estudios superiores hacia la disputa por quién, cómo, dónde, cuándo y para qué hacerlo" (Carlino, 2013, p. 355). Lo que defiende este trabajo es que si los docentes fueran capacitados en el uso de la escritura como instrumento para potenciar conocimiento se elevaría la calidad de la producción de los estudiantes, la de su propia formación y, por supuesto, la calidad del programa.

A este respecto, Chois y Jaramillo (2016) argumentan la necesidad de realizar investigaciones que indaguen más sobre la participación de los docentes en el proceso de escritura de los estudiantes y la forma en la que la estructura curricular y las tareas de escritura se articulan con la redacción de los informes, los artículos y las tesis. Por su parte, Hernández (2009) piensa que la escritura "es herramienta de aprendizaje que los estudiantes de cualquier nivel y campo disciplinario deberían saber usar. Pero debemos preguntarnos si a la educación superior y de posgrado ya no le corresponde 'enseñar a escribir'; a mi juicio sí le corresponde" (p. 36). 
Ahora, si en el escenario de la modalidad presencial lo anterior ha sido problemático, con el crecimiento de la educación virtual la tendencia podría ser el agravamiento del asunto. Es decir, la implicación importante es que el crecimiento de los posgrados que se ofertan podría no tener relación directa con el aumento de la calidad en estos (Vargas, 2016, p. 101). Además, la creciente demanda y oferta de educación virtual plantea retos en la enseñanza de escritura que, al decir de Vargas (2016), requiere cambios, por ejemplo, "en la relación con el tutor, el tipo de encuentros, su periodicidad y las diferentes etapas del proceso de escritura de la tesis. Aquí surge el interrogante sobre cómo será el proceso de asesoría de la escritura de la tesis en línea" (p. 101).

Como quiera que sea, en la modalidad presencial o virtual existen dificultades individuales e institucionales en la escritura de las tesis y los artículos de los estudiantes. Tales problemáticas se resumen en "falta de apoyo del director, dificultades personales, problemas económicos, problemas familiares, trabas por parte de la institución, otras dificultades externas" (Hirschhorn, 2012, p. 149). Pero la causa de fondo, en lo que respecta a este estudio, es el desaprovechamiento del uso epistémico de la escritura en estudiantes y en las instituciones; el asunto es que los programas, por lo general, no suelen ayudar a los universitarios a desarrollar sus capacidades escriturales como instrumento para aprender y crear y para forjar un pensamiento crítico (Carlino, 2004a). La escritura no se ha considerado - y no parece que se fuera a hacer — una actividad transversal, pues se ha relegado a una materia de primer semestre que muchas veces revisa solo actualización en asuntos gramaticales.

Tal responsabilidad institucional —que vincule de fondo la de los profesores - debe animar la del estudiante para que aprenda y practique tal enfoque de la escritura. Ello permitiría la asunción de que

$$
\begin{aligned}
& \text { escribir no es algo que deba llevarse a cabo cuando ya se han aclarado } \\
& \text { las ideas, sino justamente algo que ayuda a hacerlo, un apoyo para la } \\
& \text { actividad intelectual. No se trata de pensar para escribir, sino justamen- } \\
& \text { te de escribir para pensar. (Chois y Jaramillo, 2016, p. 228) }
\end{aligned}
$$

La escritura es un proceso que "vehicula los conocimientos, habilidades y capacidades, incluso la actitud ética del estudiante. De esto debe dar cuenta la escritura" (Cruz, 2015, p. 59). Para ello, enseñar a escribir en cada asignatura es "posibilitar que los alumnos se incluyan en situaciones discursivas típicas de comunidades especializadas, según propósitos, significados y valores compartidos" (Carlino, 2013, p. 361). Además, "puesto que la ciencia y el mundo académico se basan esencialmente en el discurso escrito, el conocimiento de cada disciplina se elabora a partir de la acumulación de los géneros escritos propios" (Cassany y Morales, 2009, p. 3). 
Frente a lo anterior, se asume que quien aplica los criterios de los diversos productos escritos "(artículo de investigación, artículo de revisión, caso clínico, memoria de grado, tesis, proyecto, etc.) está mejor preparado para leer, escribir, investigar y publicar en su disciplina, es decir, puede incorporarse con mayor facilidad a su comunidad científica" (Cassany y Morales, 2009, p. 3). La composición escrita disciplinar, sin duda, es una forma de integrar "los saberes discursivos que supone la escritura a través de los cuales se cristaliza en el texto la integración coherente y cohesiva de los saberes propios y ajenos" (Nothstein y Valente, 2016, p. 130).

Por todo ello, "Es necesario que la escritura ocupe el lugar que le corresponde, que se le reconozca como la herramienta que ofrece la oportunidad de ver con claridad las ideas y analizar su relación con las ideas de otros" (Moya et al., 2017, p. 83). En suma, que reivindique su quehacer en la escuela, donde se advierta que la competencia para la escritura no consiste en una serie de indicaciones básicas que se aprenden para siempre en los niveles iniciales de la educación (Franco, 2016, p. 156), y que no se relegue a una asignatura independiente de las demás, sino transversal en ellas. Y en lo individual, que el estudiante tenga la posibilidad de conocer y aplicar la escritura para aprender y crear.

\section{Conclusiones}

La mayoría de las percepciones que tienen los estudiantes de posgrado sobre las dificultades para escribir la tesis y el artículo científico son repetitivas si se comparan las de este estudio con otros trabajos; además, predomina el descuido de las instituciones en el acompañamiento directo al proceso de la escritura académica y sus criterios. No parece existir plena conciencia de que la calidad de los productos de los graduados dicen de la calidad de los programas académicos.

Y aunque la formación posgradual exige un nivel de competencia escritural, las prácticas de la escritura aún se orientan según la perspectiva tradicional y así "obedece más al desarrollo de contenidos académicos que a prácticas autónomas y trascendentes" (Pérez y Rincón, 2013, p. 214). Es decir, estos autores se refieren al modo tradicional de enseñanza en la que se usa la escritura más para repetir lo construido o solo como medio para plasmar lo ya pensado. Y lo que se propone aquí, es precisamente pensar en el uso de la escritura como una actividad con potencial epistémico.

Otra de las conclusiones es que la mayoría de los estudiantes e instituciones consultadas aquí desaprovechan el potencial epistémico de la escritura o no la asumen como un instrumento para aprender o fortalecer conocimiento. Esta es la mayor dificultad, cuya atención podría ayudar 
a solucionar problemas como la deserción de los estudiantes. Establecer estrategias de composición escrita que hagan parte de la asignatura para que se aproveche su poder epistémico es avanzar en herramientas para que los estudiantes aprendan mejor y edifiquen conocimiento y, por ende, no claudiquen fácilmente en su empeño académico.

En síntesis, asumir la potencia epistémica de la escritura, sin limitarse a considerarla solo como medio para expresión, es tener conciencia de que "al escribir, se trabaja sobre el pensamiento, se le da una forma entre otras posibles; la reflexión surgida a través de la escritura es diferente de la reflexión no escrita [...] al escribir se crean contenidos no existentes" (Carlino, 2005a, p. 9). En este aspecto, estudiantes, docentes e instituciones permanecen en un desaprovechamiento de la escritura como instrumento de aprendizaje y como un factor para mantener la calidad académica. Es importante anotar que, como este estudio se basa solo en cuatro universidades colombianas, no se recomienda hacer generalizaciones con base en los resultados.

\section{Referencias}

Carlino, P. (2004). El proceso de escritura académica: cuatro dificultades de la enseñanza universitaria. Educere, 8(26), 321-327.

Carlino, P. (2004a). Escribir a través del currículum: tres modelos para hacerlo en la universidad. Lectura y Vida. Revista Latinoamericana de Lectura, 25(1), 16-27.

Carlino, P. (2005a). La escritura en la investigación [Conferencia]. https:// media.utp.edu.co/referencias-bibliograficas/uploads/referencias/ponencia/255-la-escritura-en-la-investigacinpdf-v3zlj-articulo.PDF

Carlino, P. (2005b). ¿Por qué no se completan las tesis en los posgrados? Obstáculos percibidos por maestrandos en curso y magistri exitosos. Educere, 9(30), 415-420. http://www.saber.ula.ve/handle/ $123456789 / 19980$

Carlino, P. (2013). Alfabetización académica diez años después. Revista Mexicana de Investigación Educativa, 18(57), 355-381.

Cassany, D. y Morales, O. A. (2009). Leer y escribir en la universidad: los géneros científicos. En D. Cassany (Comp.), Para ser letrados. Voces y miradas sobre la lectura (pp. 109-128). Paidós.

Chois, P. y Jaramillo, L. (2016). La investigación sobre la escritura en posgrado: estado del arte. Lenguaje, 44(2), 227-259. http://www.scielo. org.co/pdf/leng/v44n2/v44n2a05.pdf

Cruz, A. G. (2015). La atención de la escritura académica en el posgrado: una experiencia en la upN. Revista de Investigación Educativa de la Rediech, 9, 51-59. http://www.redalyc.org/pdf/5216/521651961006.pdf 
Ferreyra, M. M., Avitabile, C., Botero, J., Haimovich, F. y Urzúa, S. (2017). Momento decisivo. La educación superior en América Latina y el Caribe. https://openknowledge.worldbank.org/bitstream/handle/ 10986/26489/211014ovSP.pdf? sequence=5\&isAllowed $=y$

Franco, J. (2016). Experiencia de intervención educativa para mejorar la calidad de escritura académica de los estudiantes de posgrado. Revista de Investigación Educativa 22, 151-175. http://www.scielo.org.mx/ pdf/cpue/n22/1870-5308-cpue-22-00151.pdf

Giraldo, C. (2015). La escritura en el aula como instrumento de aprendizaje. Estudio en universidades. Ánfora, 22(38), 39-58.https://doi. org/10.30854/anf.v22.n38.2015.25

Giraldo, C. (2017). Escribir y publicar artículos científicos. Sugerencias. Editorial Universidad de Manizales.

Gómez, M. Á., Deslauriers, J. P. y Alzate, M. V. (2010). Cómo hacer tesis de maestría y doctorado. ECOE Ediciones.

Hernández, G. (2009). Escritura académica y formación de maestros ¿por qué no acaban la tesis? Tiempo de Educar, 10(19), 11-40. http:// www.redalyc.org/pdf/311/31113164002.pdf

Hernández, R., Fernández, C. y Baptista, P. (2010). Metodología de la investigación (6. ${ }^{\mathrm{a}}$ ed.). McGraw-Hill.

Hirschhorn, A. N. (2012). Factores que facilitan y que dificultan la culminación de la tesis. Análisis comparado de tres escuelas de postgrado en ciencias agropecuarias (tesis de Maestría). Universidad Nacional del Mar del Plata. http://nulan.mdp.edu.ar/1625/1/hirschhorn_an.pdf

Ibáñez, C. y Marín, A. (2008). Metodologías de la investigación en las ciencias sociales: Fases, fuentes y selección de técnicas. Revista Escuela de Administración de Negocios, 64, 5-18. http://www.redalyc. org/pdf/206/20612981002.pdf

Joseph, J. (2018). It's a jungle out there: Challenges in postgraduate research writing. GEMA Online ${ }^{\circledast}$ Journal of Language Studies 18(1), 22-37. http:// doi.org/10.17576/gema-2018-1801-02

Marinkovich, J. y Moran, P. (1998). La escritura a través del currículum. Signos, 31(43-44), 165-171. https://biblat.unam.mx/fr/revista/revista-signos/7

Márquez, S., Ancira, A. M. y Lozano, A. (2013). Diagnóstico de las habilidades de redacción académica de estudiantes de nivel posgrado. Riege Investigaciones, 9-16. http://www.ruv.itesm.mx/convenio/catedra/recursos/material/re_24.pdf

Moya, C., Vanegas, I. y González, C. (2017). Escribir hoy en el posgrado. Escritura académica y producción de conocimiento. Universidad Externado de Colombia. 
Muñoz, C., Sylva, G. A. y Andrade, M. C. (2016). Manual de escritura académica. Magisterio Editorial.

Myers, L. H. (1999). Barriers to completion of the doctoral degree in Educational Administration (tesis de doctorado). https://vtechworks.lib. vt.edu/bitstream/handle/10919/26922/LHmyers.PDF?sequence=1 \&isAllowed $=y$

Narvaja, E. (Dir.) (2009). Escritura y producción de conocimiento en las carreras de posgrado. Signos. http://dx.doi.org/10.4067/S071809342013000300007, https://scielo.conicyt.cl/scielo.php?script=sci_arttext\&pid=S0718-09342013000300007

Nothstein, S. y Valente, E. (2016). La producción de escritos en postgrados internacionales. La incidencia de las tradiciones académicas en la apropiación y producción de saberes. Signos, 49(1), 127-148. https://scielo.conicyt.cl/pdf/signos/v49s1/art07.pdf

Ochoa, L. y Cueva, A. (2017). El bloqueo en el proceso de elaboración de una tesis de maestría: angustias y desazones percibidas por sus protagonistas. Lenguaje, 45(1), 61-87. http://www.scielo.org.co/pdf/ leng/v45n1/0120-3479-leng-45-01-00061.pdf

Ong, W. J. (1994). Oralidad y escritura. Tecnología de las palabras. Colombia: FCE.

Patiño, G. (2013). Escritura y universidad. Universidad del Rosario

Pérez, M. y Rincón, G. (2013). ¿Para qué se lee y se escribe en la universidad colombiana? Colciencias y Universidad Javeriana

Rodríguez, B. A. y García, L. B. (2015). Escritura de textos académicos: dificultades experimentadas por escritores noveles y sugerencias de apoyo. CPU-e, Revista de Investigación Educativa, 20, 249-265. http:// www.redalyc.org/pdf/2831/283133746012.pdf

Rodríguez, B. A. y Leal, R. A. (2016). La escritura académica en los posgrados profesionalizantes para maestros de educación básica. Revista de Investigación Educativa, 24, 224-239. http://www.scielo.org. mx/scielo.php?script=sci_arttext\&pid=S1870-53082017000100224

Scardamalia, M. y Bereiter, C. (1992). Dos modelos explicativos de los procesos de composición escrita. Infancia y Aprendizaje, 58, 43-64.

Vargas, A. (2016). La escritura académica en el posgrado: la perspectiva del estudiante. Un estudio de caso. REDU, Revista de Docencia Universitaria, 14(1), 97-129.

Zárate, M. C. (2017). La escritura académica: dificultades y necesidades en educación superior. Revista Científica Cepies, 2(1), 46-54. http:// www.scielo.org.bo/scielo.php?pid=S2518-82832017000100005\&script=sci_abstract 